observed higher concentration of antibodies a-Sa and CEP-1 than in other groups.

Antibodies a-Sa were positive in $69 \%$ of $R F(+)$ RA patients, in $37 \%$ of $R F(-) R A$ patients, in $26 \%$ of UA patients and in $10 \%$ of healthy people. In 8 aCCP (-) and $\mathrm{RF}(-)$ patients we observed the presence of a-Sa; 3 of them were diagnosed with RF(-) RA with and 5 with UA. Anti-CEP-1 antibodies were positive in $77 \%$ of RF (+) RA patients, in $56 \%$ of RF(-) RA patients, in $4,5 \%$ of UA patients, but their presence were not observed in the healthy people. In 8 aCPP (-) and RF (-) patients we observed positive anti-CEP-1 antibodies; 6 of them were diagnosed with RA, 2 of them with UA. Anti-CEP-1 antibodies were positive in $50 \%$ of RF(-) RA patients, in whom there was no aCCP nor RF, and only in $4,5 \%$ of UA patients.

In case of marking a-Car- $P$, positive values were present in: the group of $R F(+)$ RA in $40 \%$ of patients, in patients diagnosed with RF(-) RA in $6 \%$, in case of UA in $22 \%$ of patients. In patients with $\mathrm{RF}(+) \mathrm{RA}$, positive anti-CarP antibodies are present statistically significantly more often than in the group of $R F(-) R A$ patients $(p<0,05)$.

In case of marking hnRNP/RA33 and AFA no statistically significant differences between $R F(+) R A, R F(-) R A$ and UA in their occurrence were observed. In patients with arthritis no correlation between smoking and analysed autoantibodies was observed. In smokers higher CRP concentration and ESR values was observed.

Conclusions: Our results suggest that a-Sa and CEP-1 parameters allow to differentiate $R F(+) R A, R F(-) R A$ and UA, but do not differentiate UA from $R F(-) R A$. Marking CEP-1 in patients with early arthritis may help in differentiation between $\mathrm{RF}(-) \mathrm{RA}$ and UA. Marking CEP-1 and a-Sa may be useful in diagnosing early arthritis patients. The presence of Anti-CarP antibodies in UA patients is probably considerable risk factor in $R A$ development. Smoking cessation may contribute to decrease RA activity.

Disclosure of Interest: None declared

DOI: 10.1136/annrheumdis-2018-eular.5236

\section{FRI0021 PREDICTORS OF RHEUMATOID ARTHRITIS DEVELOPMENT IN PATIENTS WITH EARLY UNDIFFERENTIATED ARTHRITIS: A 2-YEARS FOLLOW- UP STUDY}

J. Molina Collada, M.G.G. Álvarez, V. Navarro-Compán, L. Nuño Nuño, A. Villalba, D. Peiteado, P. Bogas, A. Balsa. Rheumatology, Hospital Universitario La Paz, Madrid, Spain

Background: Early treatment of rheumatoid arthritis (RA) improves longterm outcomes $^{(1)}$. However, at the beginning of the disease, some patients with RA fall within undifferentiated arthritis (UA) patients. Several studies have shown that almost half of patients with UA may experience spontaneous remission ${ }^{(2)}$. So, in order to prevent overtreatment and poor outcomes, the identification of predictors of RA development is desirable.

Objectives: To determine the frequency of patients with UA evolving into RA after 2 years of follow-up and the factors contributing to predict this outcome.

Methods: A prospective analysis of an early arthritis cohort of 1377 patients from 1993 to 2017 was undertaken. For this study, 2 years follow-up data of patients who presented with UA were analysed. A detailed baseline assessment was completed including clinical features, physical examination and laboratory tests. Patients were stratified in two groups based on progression to RA (according to physician's diagnosis) or to another disease (non-RA). First, differences between groups were tested using chi-squared and Student-t tests in the univariate analysis. Second, multivariate logistic regression models were employed to investigate the association between possible predictive factors and RA development.

Results: A total of 471 UA patients were included for analysis. Mean age was $48.7 \pm 17.5$ years, $352(74.9 \%)$ were females, and mean symptoms duration was $13.9 \pm 13.9$ weeks. After 2 years of follow-up, $93(19.7 \%)$ of UA patients evolved into RA. Meanwhile, 175 (37.2\%) remained undifferentiated and 203 (43.1\%) developed into other musculoskeletal diseases. In the univariate analysis, the presence of rheumatoid factor (RF) and anti-citrullinated peptides antibodies (ACPA), tender and swollen joint count, duration of morning stiffness, smoking, symmetry and ESR values were significantly associated with RA development. In the multivariate analysis, RF $(\mathrm{OR}=5,899 ; 95 \% \mathrm{Cl} 1,795-19,382)$, ACPA $(\mathrm{OR}=123,238 ; 95 \% \mathrm{Cl} 29,353-517,410)$ and swollen joint count $(\mathrm{OR}=1,233$; $95 \% \mathrm{Cl} 1,048-1,450)$, remained significantly associated with RA development (table 1).
Abstract FRI0021 - Table 1. Independent predictors of RA development based on logistic regression model

\begin{tabular}{lccc}
\hline & \multirow{2}{*}{$\begin{array}{l}\text { Odds } \\
\text { ratio }\end{array}$} & \multicolumn{2}{c}{ 95\% C.I. } \\
\cline { 3 - 4 } & 1016 &, 983 & 1051 \\
\hline Phisician Global Asessment (0- & & & \\
100) &, 949 &, 852 & 1057 \\
28 Tender Joint Count & 1233 & 1048 & 1450 \\
28 Swollen Joint Count & 1018 &, 992 & 1044 \\
ESR(mm/h) &, 633 &, 021 & 19250 \\
Race &, 851 &, 223 & 3245 \\
Smoking &, 568 &, 145 & 2225 \\
Symmetry & 5899 & 1795 & 19382 \\
RF(IU/mL) & 123238 & 29353 & 517410 \\
ACPA(IU/mL) & & &
\end{tabular}

Conclusions: Approximately, 1 out of 5 patients with UA evolves into RA after 2 years of follow-up. Swollen joint count, and the presence of rheumatoid factor (RF) and anti-citrullinated peptides antibodies (ACPA) are independent predictors for the development of RA, supporting the early DMARDs initiation in such patients.

\section{REFERENCES:}

[1] Lard LR, Visser H, Speyer I, vander Horst-Bruinsma IE, Zwind- erman AH, Breedveld FC, et al. Early versus delayed treatment in patients with recent-onset rheumatoid arthritis: comparison of two cohorts who received different treatment strategies. Am J Med 2001;111:446-51.

[2] Olivieri I, Sarzi-Puttini P, Bugatti S, Atzeni F, d'Angelo S, Caporali R. Early treatment in early undifferentiated arthritis. Autoimmun Rev 2012;11:58992.

Disclosure of Interest: None declared

DOI: 10.1136/annrheumdis-2018-eular.3776

\section{FRI0022 RAPID AND SUSTAINED REMISSION CONTRIBUTES TO IMPROVED PSYCHOSOCIAL OUTCOMES AFTER 1 YEAR OF TREATMENT IN PATIENTS WITH EARLY RHEUMATOID ARTHRITIS: DATA FROM THE CARERA TRIAL}

K. Van der Elst ${ }^{1,2}$, P. Verschueren ${ }^{1,2}$, V. Stouten ${ }^{2}$, S. Pazmino ${ }^{2}$, D. De Cock ${ }^{2,3}$ J. Joly ${ }^{1}$, P. Moons ${ }^{4,5}$, R. Westhovens ${ }^{1,2}$. ${ }^{1}$ Rheumatology, University Hospitals Leuven; ${ }^{2}$ Skeletal Biology and Engineering Research Centre, KU Leuven, Leuven, Belgium; ${ }^{3}$ Manchester Academic Health Science Centre, University of Manchester, Manchester, UK; ${ }^{4}$ Centre for Health Services and Nursing Research, KU Leuven, Leuven, Belgium; ${ }^{5}$ Institute of Health and Care Sciences, University of Gothenburg, Gothenburg, Sweden

Background: The goal of early rheumatoid arthritis $(R A)$ treatment is to reach remission as soon as possible, as this initial treatment response is a strong predictor of long-term clinical outcomes. Rapidly reaching remission is not only clinically meaningful, it also matters from the patient's perspective. ${ }^{1}$ Yet, the influence of an initial response on patients' future psychosocial functioning remains understudied in early $R A$.

Objectives: To explore the association between the initial clinical response of patients with early RA and their psychosocial functioning after 1 year of treatment. Methods: We included patients with early RA (disease duration $\leq 1$ year) who started first-time treatment within the Care in early RA (CareRA) trial. ${ }^{2}$ Based on the speed of response defined as the response at week 16, and the stability of response evaluated from week 16 onwards until week 52 of treatment, we created 4 profiles of initial clinical response relevant to patients: persistent responders, secondary failures, delayed responders and non-responders (table 1). Having a response was defined as a disease activity score (DAS28CRP) $<2.6$ indicating remission. Psychosocial functioning was operationalized using relevant subscales of the Short-form 36 (SF-36) and the Revised Illness Perception Questionnaire (IPQ-R) (table 2). We built multiple linear regression models for each psychosocial outcome separately adjusted for confounding variables. Imputation using the Expectation-Maximisation method was performed for missing SF-36 and IPQ-R scores (range $22.2 \%>23.1 \%$ ) only at week 52 . 